March, 2012

\title{
A note on perturbation series in supersymmetric gauge theories
}

\author{
Jorge G. Russd* \\ Perimeter Institute for Theoretical Physics, Waterloo, Ontario, N2L 2Y5, Canada \\ Institució Catalana de Recerca i Estudis Avançats (ICREA) \\ Pg. Lluis Companys, 23, 08010 Barcelona, Spain
}

\begin{abstract}
Exact results in supersymmetric Chern-Simons and $\mathcal{N}=2$ Yang-Mills theories can be used to examine the quantum behavior of observables and the structure of the perturbative series. For the $U(2) \times U(2)$ ABJM model, we determine the asymptotic behavior of the perturbative series for the partition function and write it as a Borel transform. Similar results are obtained for $\mathcal{N}=2 S U(2)$ super YangMills theory with four fundamental flavors and in $\mathcal{N}=2^{*}$ super Yang-Mills theory, for the partition function as well as for the expectation values for Wilson loop and 't Hooft loop operators (in the 0 and 1 instanton sectors). In all examples, one has an alternate perturbation series where the coefficient of the $n$th term increases as $n$ !, and the perturbation series are Borel summable. We also calculate the expectation value for a Wilson loop operator in the $\mathcal{N}=2^{*} S U(N)$ theory at large $N$ in different regimes of the 't Hooft gauge coupling and mass parameter. For large masses, the calculation reproduces the running gauge coupling for the pure $\mathcal{N}=2 \mathrm{SYM}$ theory.
\end{abstract}

\footnotetext{
* On leave of absence from Universitat de Barcelona and Institute of Cosmos Sciences, Barcelona, Spain.
} 


\section{Contents}

1 Introduction $\quad 2$

2 ABJM matrix model 4

$3 \mathcal{N}=2 S U(2)$ theory with $N_{f}=4$ massless hypermultiplets 9

$4 \mathcal{N}=2^{*} S U(N)$ Super Yang-Mills theory 14

4.1 Large $N$ limit and Wilson loop . . . . . . . . . . . . . . . . . 15

$4.2 \mathcal{N}=2^{*} S U(2)$ theory . . . . . . . . . . . . . . . . . 18

4.2 .1 Wilson loop .......................... 20

4.2 .2 't Hooft loop . . . . . . . . . . . . . . . . . . . 21

5 Concluding remarks $\quad 24$

\section{Introduction}

An intriguing feature of quantum field theory is that the number of Feynman diagrams of $n$th order increases as $n$ ! [1,2]. From early times, this led to the belief that perturbation series is divergent. In some cases it may be rendered convergent by the extensively studied technique of Borel transformation [3]. If one has the series

$$
Z(g)=\sum_{n=0}^{\infty} c_{n} g^{n}
$$

by inserting a " 1 ",

$$
1=\frac{1}{n !} \int_{0}^{\infty} d t e^{-t} t^{n}
$$

and commuting integral and sum, one finds a new expression

$$
Z(g)=\int_{0}^{\infty} d t e^{-t} B(t g), \quad B(x)=\sum_{n=0}^{\infty} \frac{c_{n}}{n !} x^{n},
$$

where the expansion series for $B(x)$ has better convergence properties. In particular, if $c_{n} \sim n$ ! then $B(x)$ will have finite radius of convergence. In this case $B(x)$ will have at least one singularity somewhere in the complex (Borel) plane at some finite $x$. As long as this singularity does not lie on the real positive axes, the integral over $t$ can be performed, obtaining a finite expression for $Z(g)$ out of the original divergent expression. The trick of course lies in the illegitimate step of commuting the infinite sum and the integration; although it may appear as an artificial trick, the examples considered in this paper give a new justification in support of the Borel resummation procedure.

Some singularities of the Borel transform $B(x)$ are associated with instanton solutions [2]. The special thing about instantons is that they tend to produce singularities 
on the real axes of $B(x)$. In non-abelian gauge theories, instantons may produce singularities on the negative real axes, in such a case they are harmless. Configurations of instantons and anti-instantons of vanishing topological charge in current-current correlation functions are believed to produce singularities on the positive real axes. There can also be singularities which lie away from the real axes, and these typically correspond to (unphysical) complex field configurations. In non-abelian gauge theories the main problem in applying the Borel transform arises from renormalons [3], associated with terms in the operator product expansion. They can produce singularities on the positive real axes, leading to an ill-defined Borel integral [4]. This obstruction has been extensively studied (see e.g. [5] and references therein) leading to the theory of resurgent functions and there are prescriptions to construct well-defined quantities by deforming the contour. This gives rise to an imaginary part which in some cases describes a physical instability of the system (an example is the ground state energy of the cubic anharmonic oscillator).

In non-abelian gauge theories in four dimensions, many of these considerations are incomplete insofar as one does not know in general closed analytic expressions to all orders in the coupling for any process. In some cases, even obtaining partial results require a complicated analysis. However, the recent exact results in supersymmetric gauge theories obtained by localization techniques open the possibility of revisiting these old issues and understanding in a clean setting the origin of perturbation theory divergences.

$\mathcal{N}=2$ supersymmetric gauge theories have a rich quantum dynamics which in many respects reproduces the expected physics of QCD. The exact low energy effective action constructed by Seiberg and Witten [6,7] shows chiral symmetry breaking and quark confinement in the theory deformed by $\mathcal{N}=1$ preserving mass terms, via condensation of magnetic monopoles. More recently, exact results have been obtained for the partition function and for the expectation value of gauge invariant operators using localization [8,9]. Implementing localization requires the existence of a fermionic symmetry. One can show that the functional integral reduces to a submanifold of field configurations invariant under the fermionic symmetry that solve the saddle point equations. The complete effect of quantum fluctuations is then captured by the one-loop determinant.

In $\mathcal{N}=4 \mathrm{SYM}$ the one-loop determinant is absent and as we will see this has a striking effect on the behavior of the perturbative series. One exact result in $\mathcal{N}=4 S U(N) \mathrm{SYM}$ is the expectation value of the circular Wilson loop operator obtained by Drukker and Gross [10], extending the result of [11] beyond the planar limit. The result is expressed in terms of the following closed analytic expression:

$$
\left\langle W\left(C_{\text {circle }}\right)\right\rangle=\frac{2 e^{-\frac{g^{2}(1+N)}{8 N}}}{N ! g} \int_{0}^{\infty} d t e^{-t} t^{N-\frac{1}{2}} I_{1}(\sqrt{t} g),
$$

where $I_{1}$ is the modified Bessel function. Comparing with (1.1), we see that the integral is already given as a "Borel transform",

$$
\left\langle W\left(C_{\text {circle }}\right)\right\rangle=\frac{2 e^{-\frac{g^{2}(1+N)}{8 N}}}{N ! g^{2 N}} \int_{0}^{\infty} d t e^{-t} B\left(t g^{2}\right),
$$


where

$$
B(x) \equiv x^{N-\frac{1}{2}} I_{1}(\sqrt{x})
$$

Consider the perturbation series of $\langle W\rangle$. Using the power series expansion of Bessel function $I_{1}$, we find

$$
\begin{aligned}
\left\langle W\left(C_{\text {circle }}\right)\right\rangle & =\frac{e^{-\frac{g^{2}(1+N)}{8 N}}}{N !} \sum_{k=0}^{\infty} \frac{(N+k) ! g^{2 k}}{4^{k} k !(k+1) !} \\
& =\frac{1}{N !} \sum_{n=0}^{\infty} c_{n} g^{2 n}, \quad c_{n}=\frac{(-1)^{n}}{8^{n}} \sum_{k=0}^{n} \frac{(-1)^{k} 2^{k}(N+k) !\left(1+\frac{1}{N}\right)^{n-k}}{k !(n-k) !(k+1) !} .
\end{aligned}
$$

For large $n, c_{n} \sim b^{n} / n !, b=(N-1) / 8 N$. Thus the perturbative series for this observable has an infinite radius of convergence. As we will see this is not the case for gauge theories with less supersymmetry, where the perturbation series will be divergent.

Consider now the planar $N \rightarrow \infty$ limit, with $\lambda=g^{2} N$ fixed. Using $(N+k) ! / N ! \approx N^{k}$, we see that $\left\langle W\left(C_{\text {circle }}\right)\right\rangle \rightarrow 2 I_{1}(\sqrt{\lambda}) / \sqrt{\lambda}$, which shows that the series expansion in planar diagrams also has an infinite radius of convergence for this observable.

In this paper we consider the following theories: the ABJM theory [12], the $\mathcal{N}=2$ superconformal $S U(2)$ Yang-Mills theory with four fundamental hypermultiplets, and the $\mathcal{N}=2^{*} S U(N)$ theory obtained by adding a mass term for the adjoint hypermultiplet of $\mathcal{N}=4$ super Yang Mills (SYM) theory. Various properties of these $\mathcal{N}=2$ SYM theories are discussed in [7].

In section 2 we consider the exact expression for the partition function of $U(2) \times U(2)$ ABJM theory on $S^{3}$ obtained by Kapustin et al in [13. We determine the asymptotic behavior of the perturbation series and show that it is summable in the sense of Borel. In section 3 we show that the partition function in the zero-instanton sector of the $\mathcal{N}=2$ superconformal $S U(2)$ Yang-Mills theory on $S^{4}$ can be written as a Borel transform. This formula explicitly exhibits the singularity structure and the convergence radius of the associated series. We also determine the asymptotic behavior of the perturbation series by a saddle point evaluation and discuss the one-instanton sector. The analysis is then extended to the expectation value of a circular Wilson loop operator. Section 4 contains several calculations on the $\mathcal{N}=2^{*}$ SYM theory. We first consider the $S U(N)$ theory in the planar limit and compute the distribution of eigenvalues and the expectation value of the circular Wilson loop in different regimes of the gauge coupling and mass parameters. We then consider the $S U(2)$ gauge theory. We discuss different properties for the partition function, Wilson loop and 't Hooft loop operators, we comment on the structure and asymptotic behavior of the perturbative series, the role of instantons and on the small and large mass behavior. Section 5 contains concluding remarks.

\section{$2 \quad$ ABJM matrix model}

1. The ABJM theory [12] is constructed by using two copies of Chern-Simons theory with gauge group $U(N) \times U(N)$ and opposite levels $k,-k$. The general formula for the 
partition function of ABJM theory on $S^{3}$ is given by 13

$$
Z_{\mathrm{ABJM}}=\frac{1}{2^{2 N} N !^{2}} \int \frac{d^{N} \mu}{(2 \pi)^{N}} \frac{d^{N} \nu}{(2 \pi)^{N}} \frac{\prod_{i<j} \sinh ^{2}\left(\frac{\mu_{i}-\mu_{j}}{2}\right) \sinh ^{2}\left(\frac{\nu_{i}-\nu_{j}}{2}\right)}{\prod_{i, j} \cosh ^{2}\left(\frac{\mu_{i}-\nu_{j}}{2}\right)} e^{\frac{i k}{4 \pi} \sum_{i=1}^{N}\left(\mu_{i}^{2}-\nu_{i}^{2}\right)} .
$$

$1 / k$ plays the role of coupling constant. By some simple manipulations the partition function can be casted in the following form [14, 15]

$$
Z_{\mathrm{ABJM}}=\frac{1}{2^{2 N} N !} \sum_{\sigma \in S_{N}}(-1)^{\varepsilon(\sigma)} \int \frac{d^{N} x}{(2 \pi k)^{N}} \frac{1}{\prod_{i} \cosh \left(\frac{x_{i}}{2}\right) \cosh \left(\frac{x_{i}-x_{\sigma(i)}}{2 k}\right)} .
$$

Different aspects of the partition function have also been discussed in [16, 17].

We begin the discussion with a case where calculations can be made explicitly. The ABJM model with gauge group $U(2) \times U(2)$, i.e. $N=2$. In this case we have

$$
\begin{aligned}
Z_{\mathrm{ABJM}}(N=2) & =\frac{1}{32} \int \frac{d x_{1} d x_{2}}{(2 \pi k)^{2}} \frac{1}{\cosh \left(\frac{x_{1}}{2}\right) \cosh \left(\frac{x_{2}}{2}\right)}\left(1-\frac{1}{\cosh ^{2}\left(\frac{x_{1}-x_{2}}{2 k}\right)}\right) \\
& =\frac{1}{32 k^{2}}(1-J(k)),
\end{aligned}
$$

where

$$
J(k) \equiv \frac{1}{4 \pi^{2}} \int d x_{1} d x_{2} \frac{1}{\cosh \left(\frac{x_{1}}{2}\right) \cosh \left(\frac{x_{2}}{2}\right) \cosh ^{2}\left(\frac{x_{1}-x_{2}}{2 k}\right)} .
$$

Using the Fourier integrals:

$$
\begin{aligned}
& \frac{1}{\cosh ^{2}\left(\frac{x_{1}-x_{2}}{2 k}\right)}=2 k^{2} \int d t \frac{t}{\sinh (\pi k t)} e^{i t\left(x_{1}-x_{2}\right)} \\
& \int d x \frac{1}{\cosh \frac{x}{2}} e^{i t x}=\frac{2 \pi}{\cosh (\pi t)}
\end{aligned}
$$

we can perform the integrals over $x_{1}, x_{2}$. We get

$$
J(k)=\frac{2}{\pi^{2}} \int d v \frac{1}{\cosh ^{2}\left(\frac{v}{k}\right)} \frac{v}{\sinh (v)}, \quad v=\pi t k .
$$

This integral can be evaluated by residues, as done in [18]. The result is a finite sum of $k$ different contributions of $k$-depending terms. For the purpose of studying the power series expansion in $1 / k$, it is more convenient to work with the integral representation (2.6) of $J(k)$. We introduce

$$
I(k) \equiv-\frac{\pi^{2}}{2} \int \frac{d k}{k^{2}} J(k)=\int d v \frac{\tanh \left(\frac{v}{k}\right)}{\sinh (v)} .
$$


Using the formulas

$$
\begin{aligned}
& \tanh \left(\frac{v}{k}\right)=\sum_{m=1}^{\infty} 4^{m}\left(4^{m}-1\right) \frac{B_{2 m}}{(2 m) !}\left(\frac{v}{k}\right)^{2 m-1} \\
& \int_{-\infty}^{\infty} d v \frac{v^{2 m-1}}{\sinh (v)}=(-1)^{m+1} \frac{1}{m} \pi^{2 m}\left(4^{m}-1\right) B_{2 m},
\end{aligned}
$$

where $B_{2 n}$ are the Bernoulli numbers, we find

$$
I(k)=-k \sum_{m=1}^{\infty}(-1)^{m} \frac{\left(B_{2 m}\right)^{2}}{m(2 m) !}\left(4^{m}-1\right)^{2}\left(\frac{2 \pi}{k}\right)^{2 m} .
$$

Hence

$$
J(k)=-\frac{2 k^{2}}{\pi^{2}} I^{\prime}(k)=-\frac{2 k^{2}}{\pi^{2}} \sum_{m=1}^{\infty}(-1)^{m}(2 m-1)\left(4^{m}-1\right)^{2} \frac{\left(B_{2 m}\right)^{2}}{m(2 m) !}\left(\frac{2 \pi}{k}\right)^{2 m},
$$

and

$$
\begin{aligned}
Z_{\mathrm{ABJM}}(N=2, k) & =\frac{2}{(4 \pi)^{2}} \sum_{m=2}^{\infty}(-1)^{m} \frac{(2 m-1)}{2 m}\left(4^{m}-1\right)^{2} \frac{\left(B_{2 m}\right)^{2}}{(2 m) !}\left(\frac{2 \pi}{k}\right)^{2 m} \\
& =\frac{\pi^{2}}{64 k^{4}}\left(1-\frac{4 \pi^{2}}{3 k^{2}}+\frac{289 \pi^{4}}{90 k^{4}}-\frac{3844 \pi^{6}}{315 k^{6}}+\ldots\right) .
\end{aligned}
$$

It is interesting to examine the asymptotic behavior of this series. We use the familiar formula:

$$
\begin{aligned}
\frac{B_{2 m}}{(2 m) !} & =(-1)^{m+1} \frac{2}{(2 \pi)^{2 m}} \zeta(2 m) \\
& =(-1)^{m+1} \frac{2}{(2 \pi)^{2 m}}\left(1+2^{-2 m}+3^{-2 m}+4^{-2 m}+\ldots\right),
\end{aligned}
$$

We find the following behavior:

$$
Z_{\mathrm{ABJM}}(N=2) \sim \sum_{m \gg 1}(-1)^{m}(2 m) !\left(\frac{2}{\pi k}\right)^{2 m} .
$$

Thus the coefficient of $1 / k^{n}$ increases as $n$ !. The series (2.12) is therefore an asymptotic series in the sense of Poincaré. As more terms of the series are included, the sum approaches the true value of $Z_{\mathrm{ABJM}}(N=2, k)$ until it begins to deviate leading to a divergent asymptotic behavior. Minimizing a generic term with respect to $m$ one finds the optimal truncation (see discussion at the end of section 3).

The series is divergent and at this point the main question is whether it is Borel summable. The associated Borel series can be defined in the standard way: one introduces a "1",

$$
1=\frac{1}{(2 m) !} \int_{0}^{\infty} d t t^{2 m} e^{-t}
$$


and write

$$
Z_{\mathrm{ABJM}}(N=2, k)=\frac{2}{(4 \pi)^{2}} \sum_{n=2}^{\infty} \int d t e^{-t}(-1)^{m} \frac{(2 m-1)}{2 m}\left(4^{m}-1\right)^{2} \frac{\left(B_{2 m}\right)^{2}}{(2 m) !^{2}}\left(\frac{2 \pi t}{k}\right)^{2 m}
$$

Because the series is divergent, commuting integral and sum does not give an equivalent result. Instead it defines the associated Borel series $B(t)$,

$$
\begin{gathered}
\hat{Z}_{\mathrm{ABJM}}(N=2, k) \equiv \int_{0}^{\infty} d t e^{-t} B(t / k) \\
B(t) \equiv \frac{2}{(4 \pi)^{2}} \sum_{n=2}^{\infty}(-1)^{m} \frac{(2 m-1)}{2 m}\left(4^{m}-1\right)^{2} \frac{\left(B_{2 m}\right)^{2}}{(2 m) !^{2}}(2 \pi t)^{2 m} .
\end{gathered}
$$

The perturbation series will be Borel summable if the integral (2.17) can be done, which requires that possible singularities in $B(t)$ do not lie on the positive real $t$ axes (or, if they do, they are integrable, like e.g. in the case of a Dirac delta function singularity). Asymptotically, $B(t)$ behaves as follows

$$
B(t) \sim \sum_{m \gg 1}(-1)^{m}\left(\frac{2 t}{\pi}\right)^{2 m}
$$

which has a finite radius of convergence. More precisely, inserting the full expansion (2.13), we find that $B(t)$ is a sum of series with finite radii of convergence, the minimum radius being at $R_{*}=\frac{\pi}{2}$ so that all series are convergent for $|t|<R_{*}$. Other series have radius of convergence of the form $R=\frac{p \pi}{2}$, where $p$ is a positive integer. There are singularities in $B(t)$ on the imaginary axes at $t= \pm \frac{i \pi}{2}$ and more generally at $t=\frac{i \pi p}{2}$ where $p \in \mathbf{Z}$.

Therefore we have shown that the perturbation series for the $U(2) \times U(2)$ ABJM partition function is Borel summable. This is an expected result, because there are no instantons in three dimensions. We recall that a singularity of $B(t / k)$ on the real positive axes of $t$ at $t / k=a$ would have implied the presence of euclidean solutions with finite action $e^{-t} \sim e^{-a k}$.

2. We now consider an alternative treatment. In the present case, having closed analytic expressions, there is actually no need of artificially introducing a Borel associated sum by a Laplace transform. Indeed, one may define a slight modification of the Borel transform that works equally well and is more convenient in cases where the one-loop determinants involve hyperbolic trigonometric functions. Given an asymptotic series

$$
F(g)=\sum_{n=0}^{\infty} f_{n} g^{n}
$$

one can introduce an associated series $\mathcal{S}(z)$ defined by

$$
F(g)=2 \int_{0}^{\infty} d z \frac{z}{\sinh z} \mathcal{S}(g z)
$$


with the expansion

$$
\mathcal{S}(x)=\sum_{n=0}^{\infty} s_{n} x^{n},
$$

where $s_{n}$ is related to the original series coefficient $f_{n}$ by the formula

$$
f_{n}=2^{-n}\left(2^{n+2}-1\right) \zeta(n+2)(n+1) ! s_{n} .
$$

Here we have used the formula

$$
2 \int_{0}^{\infty} d v \frac{v^{n+1}}{\sinh (v)}=2^{-n}\left(2^{n+2}-1\right) \zeta(n+2)(n+1) ! .
$$

Asymptotically, $\zeta(n) \rightarrow 1$, therefore

$$
f_{n} \sim(n+1) ! s_{n}
$$

like in the Borel case. Thus the transformation (2.21) has essentially the same effect of the standard Borel transform. In the present $U(2) \times U(2)$ case, we do not know how to express $Z_{\mathrm{ABJM}}$ as a Borel transform in terms of closed analytic formulas, but we can express $Z_{\mathrm{ABJM}}$ in terms of the above $\mathcal{S}$-transform (2.21). This has some obvious advantages. In particular, by (2.21), one readily spots an associated series with finite radius of convergence, whose expression is determined by a closed analytic formula:

$$
Z_{\mathrm{ABJM}}(N=2)=\frac{1}{8 \pi^{2} k^{2}} \int_{0}^{\infty} d v \frac{v}{\sinh (v)} \mathcal{S}\left(\frac{v}{k}\right)
$$

with

$$
\mathcal{S}(x) \equiv \tanh ^{2}(x)
$$

By means of the sole structure of $\mathcal{S}$ one determines that:

a) singularities of $\mathcal{S}$ arise at complex values for $v, v=i k(2 n+1) \frac{\pi}{2}$ with integer $n$.

b) the series expansion of $\mathcal{S}$ in powers of $1 / k$ has a finite radius of convergence $\frac{1}{|k|}<\frac{\pi}{2}$.

c) the perturbation series for the partition function has an asymptotic behavior $(2 n)$ ! for a term $k^{-2 n}$.

d) the perturbation series is summable as the poles of the integrand do not occur at the positive real axes.

3. Consider now the general $U(N) \times U(N)$ case. A way to understand the expected asymptotic behavior of the perturbative series is by a closer look at the partition function (2.1). For this, it is convenient to write down the hyperbolic cosines in the familiar infinite product representation,

$$
\cosh x=\prod_{n=1}^{\infty}\left(1+\frac{4 x^{2}}{\pi^{2}(2 n-1)^{2}}\right)
$$


The perturbation series arises in expanding the integrand (except the exponential factor) in powers series of $\mu_{i}, \nu_{i}$ and then integrating over $\mu_{i}, \nu_{i}$ (again this involves an illegitimate commutation of sums and integrals). The series for the integrand have finite radii of convergence determined by the singularities at $\mu_{i}=\nu_{j}+i(2 l+1) \pi$ where the cosh factors in the denominator vanish. Each integral over $d \mu_{i}$ or $d \nu_{i}$ of given factors $\mu_{i}^{2 n_{i}}, \nu_{i}^{2 m_{i}}$ times the exponential factor in (2.1) introduce factorials $n_{i} !, m_{i}$ ! which will lead to a divergent behavior of the perturbative series. The fact that the final divergent perturbative series is summable is obvious because it originates from the integral in (2.1), which is well defined and convergent, in particular, it does not hit any of the singularities in the complex plane. A direct way to determine the asymptotic behavior of the perturbative series is by means of saddle-point techniques. Defining $\kappa=1 / k^{2}$, the coefficient $d_{n}$ of the $n$-th term $\kappa^{n}$ of the power series in $\kappa$ is obtained by

$$
d_{n}=\frac{1}{2 \pi i} \oint \frac{d \kappa}{\kappa^{n+1}} Z_{\mathrm{ABJM}}(N, \kappa),
$$

where the contour surrounds the point $\kappa=0$. For large $n$, the integrals over $\kappa$ and over $\mu_{i}, \nu_{i}$ are dominated by saddle points in the complex plane, e.g. $\mu_{i}=\nu_{j}+i(2 l+1) \pi+$ $O(1 / n)$, etc. We will employ this method in discussing the perturbative series for $\mathcal{N}=2$ super Yang-Mills theories.

\section{$3 \mathcal{N}=2 S U(2)$ theory with $N_{f}=4$ massless hypermul- tiplets}

Consider first $\mathcal{N}=2 S U(N)$ theory with $\mathcal{N}_{f}=2 N$ fundamental massless flavors. This theory has superconformal symmetry. The partition function on $S^{4}$ is given by [8]

$$
Z_{\mathrm{SCF}}=c_{N} g^{-\left(N^{2}-1\right)} \int d^{N-1} a \prod_{i<j}\left(a_{i}-a_{j}\right)^{2} e^{-\frac{8 \pi^{2}}{g^{2}} \sum_{i} a_{i}^{2}} Z_{1-\mathrm{loop}}(a)\left|Z_{\text {inst }}\left(a, g^{2}\right)\right|^{2},
$$

where

$$
Z_{1-\mathrm{loop}}(a)=\frac{\prod_{i<j} H^{2}\left(a_{i}-a_{j}\right)}{\prod_{i} H^{2 N}\left(a_{i}\right)}, \quad H(x) \equiv \prod_{n=1}^{\infty}\left(1+\frac{x^{2}}{n^{2}}\right)^{n} e^{-\frac{x^{2}}{n}},
$$

and $\sum_{i} a_{i}=0 . c_{N}$ is a numerical constant independent of $g$. Following [24], we introduced the function $H$ related to the Barnes $G$ function by the relation

$$
H(x)=e^{-(1+\gamma) x^{2}} G(1+i x) G(1-i x),
$$

where $\gamma$ is the Euler's constant. The instanton factor has a more complicated form and general formulas are given in [19 21]. For the case of $S^{4}$, one must fix the Nekrasov deformation parameters [19] to $\varepsilon_{1}=\varepsilon_{2}=1 / r$, where $r$ is the radius of $S^{4}$ [22]. In what follows we set $r=1$. 
Various properties of the partition function and circular Wilson loop for the large $N$ $S U(N)$ theory have been recently discussed in [23, 24]. In this section we focus on the $S U(2)$ theory. For an $S U(2)$ gauge group, the partition function reduces to

$$
Z_{\mathrm{SCF}}^{S U(2)}=128 \pi^{5 / 2} g^{-3} \int_{-\infty}^{\infty} d a a^{2} e^{-\frac{16 \pi^{2}}{g^{2}} a^{2}} \prod_{n=1}^{\infty} \frac{\left(1+\frac{4 a^{2}}{n^{2}}\right)^{2 n}}{\left(1+\frac{a^{2}}{n^{2}}\right)^{8 n}}\left|Z_{\mathrm{inst}}^{S U(2)}\right|^{2},
$$

where we have fixed the numerical constant $c_{N}$ to have $Z_{\mathrm{SCF}}^{S U(2)}=1$ at $g=0$. Now

$$
\prod_{n=1}^{\infty} \frac{\left(1+\frac{4 a^{2}}{n^{2}}\right)^{2 n}}{\left(1+\frac{a^{2}}{n^{2}}\right)^{8 n}}=\frac{H^{2}(2 a)}{H^{8}(a)}=\frac{|G(1+i 2 a)|^{4}}{|G(1+i a)|^{16}} .
$$

Consider the $(k=0)$-instanton sector, obtained by setting $\left|Z_{\text {inst }}^{S U(2)}\right| \rightarrow 1$ in (3.4). By the change of variable

$$
a^{2}=\alpha t, \quad \alpha \equiv \frac{g^{2}}{16 \pi^{2}}
$$

we get

$$
\left.Z_{\mathrm{SCF}}^{S U(2)}\right|_{k=0}=\frac{2}{\sqrt{\pi \alpha}} \int_{0}^{\infty} d t e^{-t} B(\alpha t)
$$

where

$$
B(x)=\sqrt{x} \prod_{n=1}^{\infty} \frac{\left(1+\frac{4 x}{n^{2}}\right)^{2 n}}{\left(1+\frac{x}{n^{2}}\right)^{8 n}} .
$$

Remarkably, in the zero instanton sector the partition function is already in the form of a Laplace transform of a Borel associated function.

This compact form of $B(x)$ explicitly exhibits the singularities in the Borel plane. There are singularities at

$$
\alpha t_{s}=-n^{2}, \quad n=1,2, \ldots
$$

The expansion in powers of $\alpha t$ of $B(\alpha t)$ has a finite radius of convergence given by $\left|\alpha t_{*}\right|=$ 1. Integrating over $t$ adds a factor $n$ ! to each $n$-th term $\alpha^{n}$ of this expansion. Since the series for $B(\alpha t)$ has a finite radius of convergence, the $n$-th term of the perturbative series will have the asymptotic behavior $n$ ! (more precisely, $(-1)^{n} n$ ! since the singularity appears at a negative $t$ ).

It is easy to compute the first few terms of the perturbative expansion. Using

$$
B(x)=\sqrt{x} \exp \left[-2 \sum_{k=2}^{\infty} \frac{(-1)^{k}}{k} x^{k} \zeta(2 k-1)\left(2^{2 k}-4\right)\right],
$$

we find

$$
\begin{aligned}
\left.Z_{\mathrm{SCF}}^{S U(2)}\right|_{k=0} & =1-45 \alpha^{2} \zeta(3)+525 \alpha^{3} \zeta(5)+\frac{8505}{8} \alpha^{4}\left(4 \zeta(3)^{2}-7 \zeta(7)\right) \\
& -\frac{31185}{4} \alpha^{5}(20 \zeta(3) \zeta(5)-17 \zeta(9)) \\
& -\frac{135135}{16} \alpha^{6}\left(72 \zeta(3)^{3}-200 \zeta(5)^{2}-378 \zeta(3) \zeta(7)+341 \zeta(11)\right)+O\left(\alpha^{7}\right) .
\end{aligned}
$$


Note that no $\pi$ factors appear in the perturbative expansion; it is an expansion in powers of $\alpha=g^{2} / 16 \pi^{2}$ and the associated Borel sum is convergent for $|\alpha t|<1$. The partition function is well defined for any $\alpha$ as there are no singularities in the integration region.

The divergent perturbative series (3.11) is what one would have obtained if instead of using matrix model localization techniques the partition function had been computed using Feynman diagrams 1 In the present context, it is clear that the divergence of the perturbative series emerges artificially, in the process of commuting the integral over $t$ with the sum defining the expansion of $B$ in powers of $\alpha t$. There is no divergence in the original integral defining the partition function. The singularities of $B(\alpha t)$ lie on the negative real axes, which means that the integral over $t$ can be performed and therefore the divergent perturbative series computed by Feynman diagrams is summable in the Borel sense. It is the Feynman diagrammatic method which introduces the divergent behavior, because computing Feynman diagrams requires commuting the functional integral with the expansion in series of the exponential of the interaction terms. The convergent Borel associated sum is indeed the true result of the theory, as defined by the functional integration; the divergent perturbative series here (and in the Feynman diagram calculation) arises artificially due to a mathematically illegitimate manipulation.

Let us now compute the asymptotic behavior of the perturbative series by a saddle point calculation at large $n$. Writing

$$
\left.Z_{\mathrm{SCF}}^{S U(2)}\right|_{k=0}=\sum_{n=0}^{\infty} d_{n} \alpha^{n}
$$

we have

$$
\begin{gathered}
d_{n}=\left.\frac{1}{2 \pi i} \oint \frac{d \alpha}{\alpha^{n+1}} Z_{\mathrm{SYM}}^{S U(2)}\right|_{k=0}=\frac{1}{2 \pi i} \frac{2}{\sqrt{\pi \alpha}} \oint d \alpha \int_{0}^{\infty} d t e^{-S}, \\
S(\alpha, t)=t+(n+1) \log \alpha-\log B(\alpha t) .
\end{gathered}
$$

For large $n$, the saddle point equations in $\alpha$ and $t$ are

$$
\frac{n}{\alpha}=\frac{t B^{\prime}(\alpha t)}{B(\alpha t)}, \quad 1=\frac{\alpha B^{\prime}(\alpha t)}{B(\alpha t)},
$$

i.e. $t_{*}=n$ and $\alpha$ satisfying the equation $\alpha B^{\prime}(\alpha n) / B(\alpha n)=1$. This equation has an infinite number of solutions, with $\alpha n$ near the zeroes and singularities of $B(\alpha n)$. The dominant saddle point (with smallest $S$ and large eigenvalues of the Jacobian matrix), appears near the first pole, $\alpha_{*} n=-1+O(1 / n)$. Computing $S$ at this point, we find

$$
d_{n} \sim e^{-S\left(\alpha_{*}, t_{*}\right)} \sim(-1)^{n} n^{n} e^{-n} \sim(-1)^{n} n !
$$

as anticipated above.

\footnotetext{
${ }^{1}$ For example, the three-loop term with $\zeta(3)$ coefficient in the similar perturbative series for the Wilson loop, see (3.26), has been reproduced by computing three loop Feynman diagrams in [25].
} 
It is well known that the existence of instantons can lead to singularities in the Borel transform. Having closed analytic expressions, it is therefore interesting to study how the perturbation series behave in the non-zero instanton sectors and to determine the extent to which possible singularities produced by instantons could affect the convergence of integrals. Consider for concreteness the one-instanton sector. The one-instanton contribution is given by

$$
\begin{aligned}
Z_{\text {one-inst }}^{S U(2)} & =4 e^{-\frac{8 \pi^{2}}{g^{2}}} \int \frac{d y}{2 \pi}\left(\frac{y^{4}}{\left((y-a)^{2}+1\right)\left((y+a)^{2}+1\right)}-1\right) \\
& =e^{-\frac{8 \pi^{2}}{g^{2}}}\left(a^{2}-3\right) .
\end{aligned}
$$

Thus

$$
\left.Z_{\mathrm{SCF}}^{S U(2)}\right|_{k=1}=\int_{-\infty}^{\infty} d a a^{2} e^{-\frac{\left(a^{2}+1\right)}{\alpha}}\left(a^{2}-3\right)^{2} \frac{H^{2}(2 a)}{H^{8}(a)} .
$$

This integral is well defined and convergent. Now the partition function is no longer in the form of a Laplace transform of a Borel function, because of the presence of the instanton weight $e^{-\frac{|k|}{\alpha}}$. To write it as a Borel transform one would need to put it in the form

$$
\left.Z_{\mathrm{SCF}}^{S U(2)}\right|_{k=1}=\int_{0}^{\infty} d z e^{-\frac{z}{\alpha}} F(z)
$$

This can be done by a change of coordinate, $a^{2}+1=z$. We find

$$
F(z) \equiv \theta(z-1) \sqrt{z-1}(z-4)^{2} f(z-1) .
$$

This has a branch cut singularity at $z=1$ (more generally, $z=|k|$ in the $|k|$ instanton sector), but obviously it does not affect the convergence of the integral. Let us now see how this singularity is manifested in the saddle-point evaluation. The analysis is as follows. In the 1-instanton sector, instead of (3.14) we would have

$$
S(\alpha, a)=\frac{a^{2}}{\alpha}+n \log \alpha-2 \log \frac{H(2 a)}{H^{4}(a)}+\frac{1}{\alpha}-\log a^{2}-\log \left(a^{2}-3\right)^{2} .
$$

The saddle points which lie near the singularities of $\log H(2 a) / H^{4}(a)$ are not significantly modified. The main difference is that now a new saddle point emerges, located at $a_{*}^{2}=$ $\alpha_{*}=1 / n$. Since $a^{2} \sim 0$, terms $\log H(2 a) / H^{4}(a)$ and $\log \left(a^{2}-3\right)^{2}$ are irrelevant. This is indeed the dominant saddle point. The corresponding saddle point in the $|k|$ instanton sector is located at $a_{*}^{2}=\alpha_{*}=|k| / n$. One finds

$$
d_{n} \sim \frac{n^{n}}{|k|^{n}} e^{-n} \sim n !|k|^{-n}
$$

which shows that the Borel function must have a singularity at $z=|k|$. This is precisely the location of the branch-cut singularity.

In conclusion, the integral defining the partition function is convergent in the oneinstanton sector. The instanton produces the expected singularity in the Borel function, which in the present case turns out to be harmless. 


\section{Wilson loop}

Similar considerations can be made for physical observables such as expectation values of Wilson loop operators. Do they exhibit the same asymptotic behavior? We restrict to the zero-instanton sector. For a circular Wilson loop located at the equator of $S^{4}$ in the spin- $j$ representation, one has [8]

$$
\left\langle\operatorname{tr}_{j} \operatorname{Pexp}\left(\int A d x+i \Phi_{0} d s\right)\right\rangle=Z^{-1} \int_{-\infty}^{\infty} d a a^{2} e^{-\frac{16 \pi^{2}}{g^{2}} a^{2}} \prod_{n=1}^{\infty} \frac{\left(1+\frac{4 a^{2}}{n^{2}}\right)^{2 n}}{\left(1+\frac{a^{2}}{n^{2}}\right)^{8 n}}\left(\sum_{\ell=-j}^{j} e^{4 \pi \ell a}\right) .
$$

Consider the term $e^{4 \pi \ell a}(\ell$ can be integer or half-integer). We have

$$
\left\langle e^{4 \pi \ell a}\right\rangle=\frac{\int_{0}^{\infty} d t e^{-t} B(\alpha t) \cosh (4 \pi \ell \sqrt{\alpha t})}{\int_{0}^{\infty} d t e^{-t} B(\alpha t)} .
$$

This can be expanded in powers of $\alpha$ as done in [8]. We find

$$
\begin{aligned}
\left\langle e^{4 \pi \ell a}\right\rangle & =1+12 \pi^{2} \alpha \ell^{2}+40 \pi^{4} \alpha^{2} \ell^{4}+\alpha^{3}\left(\frac{224 \pi^{6} \ell^{6}}{3}-720 \pi^{2} l^{2} \zeta(3)\right) \\
& +\alpha^{4}\left(96 \pi^{8} \ell^{8}-5760 \pi^{4} \ell^{4} \zeta(3)+12600 \pi^{2} \ell^{2} \zeta(5)\right)+O\left(\alpha^{5}\right)
\end{aligned}
$$

This expression has been checked up to the three-loop $\alpha^{3}$ order in [25] by explicit evaluation of the Feynman diagrams in perturbation theory.

A term with a given power $p$ of $\alpha$ is multiplied by a polynomial in $\ell$ of degree $2 p$. Terms with lower power of $\ell$ have coefficients which increase more rapidly with $p$. This is because each power $\ell^{2 s} t^{s}$ is accompanied by a factor $1 /(2 s)$ ! coming from the power expansion of $\cosh (4 \pi \ell \sqrt{\alpha t})$. Thus the terms in the perturbative series for the Wilson loop that give rise to the most divergent behavior of the series are those with lower power of $\ell$ in the polynomial of degree $2 p$ multiplying $\alpha^{p}$. For them, $s=1$, so such terms clearly present the same asymptotic behavior $(-1)^{n} n$ ! that appeared in $Z_{\mathrm{SCF}}^{S U(2)}$.

A different behavior can be found in a limit where $\ell \rightarrow \infty$ and $\alpha \rightarrow 0$ with $\sqrt{\alpha} \ell$ fixed. This gives

$$
\begin{aligned}
\left\langle e^{4 \pi \ell a}\right\rangle & \rightarrow \frac{\int_{0}^{\infty} d t e^{-t} \sqrt{\alpha t} \cosh (4 \pi \ell \sqrt{\alpha t})}{\int_{0}^{\infty} d t e^{-t} \sqrt{\alpha t}} \\
& =e^{4 \pi^{2} \alpha \ell^{2}}\left(1+8 \pi^{2} \alpha \ell^{2}\right) \\
& =e^{\frac{g^{2} \ell^{2}}{4}}\left(1+\frac{g^{2} \ell^{2}}{2}\right) .
\end{aligned}
$$

In this limit the one-loop determinant was set to $1, B(\alpha t) \rightarrow \sqrt{\alpha t}$. The result must then be the same as in $\mathcal{N}=4 \mathrm{SYM}$ as described in the introduction. Indeed, by explicitly computing the integral (1.2) with $N=2$, we exactly reproduce (3.27) with $\ell=1 / 2$. Now the expansion in powers of $\alpha$ has an infinite radius of convergence and $\left\langle e^{4 \pi \ell a}\right\rangle$ is an exponentially increasing function of the parameter $\alpha \ell^{2}$ which starts at 1 . 
An interesting question regards the best resolution that the asymptotic perturbative series defining the Wilson loop can provide. This is done in the standard way by considering the partial sum

$$
W_{n_{0}}=\sum_{n=1}^{n_{0}} c_{n} \alpha^{n}, \quad c_{n} \sim(-1)^{n} n ! R^{-n} .
$$

The optimal truncation is then obtained by using the Stirling approximation and minimizing $\left|c_{n}\right| \sim n ! R^{-n}$ with respect to $n$. One finds $n_{0}=R / \alpha$, for which the best resolution (or "non-perturbative ambiguity") is $\epsilon=\sqrt{2 \pi R / \alpha} e^{-R / \alpha}$, where we used the Stirling formula. In the present case, we have closed expressions which allow an accurate determination of $R$, which is nothing but the radius of convergence of the associated Borel series. This gives $R=1$, so that $n_{0}=16 \pi^{2} / g^{2}$ and the best resolution is $\epsilon=\sqrt{2 \pi / \alpha} e^{-1 / \alpha} \sim e^{-16 \pi^{2} / g^{2}}$. For example, if $g=1$, then $\alpha \sim 0.006$; this gives $n_{0}=158$ and $\epsilon=10^{-67}$. But if $g=8$, $\alpha \sim 0.4$, then $n_{0} \sim 2.4$ and the asymptotic formulas are not applicable. It should be

noted that here the "non-perturbative ambiguity" $\epsilon=\sqrt{2 \pi R / \alpha} e^{-R / \alpha}$ is unrelated to instanton contributions that would provide a "completion" of the series, since the origin of the divergence in the asymptotic series are singularities in the one-loop determinant. In particular, in the ABJM model discussed in section 2 there is a similar non-perturbative ambiguity $\epsilon=O\left(e^{-\frac{\pi k}{2}}\right)$ despite the fact that there are no instantons.

\section{$4 \mathcal{N}=2^{*} S U(N)$ Super Yang-Mills theory}

It is of interest to consider the behavior of the perturbative series and of loop operators in a case where there is no superconformal invariance. Typically, in such cases the one-loop determinant contains a divergence which needs to be renormalized [8]. One case where this divergence is innocuous is in the $\mathcal{N}=2^{*} S U(N)$ SYM, obtained by deformation of $\mathcal{N}=4$ by adding a mass $M$ for the adjoint hypermultiplet. In this case the divergence is a numerical factor (in particular, it has no incidence on the expectation value of physical observables). We remove this divergent numerical factor from the partition function by inserting $e^{-\frac{2 M^{2}}{n}}$ in the infinite product over $n$ in the integrand. The partition function on $S^{4}$ reads [8]

$$
Z^{N=2^{*}}=\int d^{N-1} a \prod_{i<j}\left(a_{i}-a_{j}\right)^{2} e^{-\frac{8 \pi^{2}}{g^{2}} \sum_{i} a_{i}^{2}} Z_{1-\mathrm{loop}}(a, M)\left|Z_{\mathrm{inst}}^{S U(N)}\right|^{2},
$$

with

$$
\begin{aligned}
Z_{1-\mathrm{loop}}(a, M) & =\prod_{i<j} \frac{H^{2}\left(a_{i}-a_{j}\right)}{H^{2}\left(a_{i}-a_{j}-M\right)} \\
& =\prod_{i<j} \prod_{n=1}^{\infty} \frac{\left(1+\frac{\left(a_{i}-a_{j}\right)^{2}}{n^{2}}\right)^{2 n}}{\left(1+\frac{\left(a_{i}-a_{j}-M\right)^{2}}{n^{2}}\right)^{n}\left(1+\frac{\left(a_{i}-a_{j}+M\right)^{2}}{n^{2}}\right)^{n} e^{-\frac{2 M^{2}}{n}}} .
\end{aligned}
$$


$\mathcal{N}=2^{*} S U(N)$ super Yang-Mills theory interpolates between $\mathcal{N}=4 S U(N)$ SYM and pure $\mathcal{N}=2 S U(N)$ SYM as the mass parameter is varied from zero to infinity. The interpolation seems to be smooth (for example, on $\mathbf{R}^{4}$ the Seiberg-Witten curve presents no discontinuous or singular behavior for any $M$ [7]). For large $M$, the $\mathcal{N}=2^{*}$ theory may be viewed as a regulated version of pure $\mathcal{N}=2$ theory where one has an UV cutoff determined by the mass. More generally, given an asymptotically free $\mathcal{N}=2$ theory, by adding a sufficient number of massive hypermultiplets one gets a theory that is well defined in the UV and for large masses it provides a regulated version of the $\mathcal{N}=2$ theory that one wishes to study. This interpretation was exploited in [8] to recover the $\beta$ function of $\mathcal{N}=2 \mathrm{SYM}$ from the partition function. This observation will be reproduced here in the planar limit. In addition, we will also study the small mass behavior and loop operators in the $S U(2)$ theory.

\subsection{Large $N$ limit and Wilson loop}

We consider planar limit, i.e. $N \rightarrow \infty$ with $\lambda=g^{2} N$ fixed. In this limit $\left|Z_{\text {inst }}^{S U(N)}\right| \rightarrow 1$. We will compute the integral in the saddle point approximation. We use the notation of [24], where the similar calculation was carried out for the $\mathcal{N}=2$ superconformal theory discussed in the previous section. Our approach to solve the system is different: now there is an extra parameter, the mass, in addition to the 't Hooft coupling $\lambda=g^{2} N$, which will lead us to consider different regimes.

The effective action for the eigenvalues is

$$
S(a)=\sum_{i} \frac{8 \pi^{2}}{\lambda} a_{i}^{2}-\frac{1}{N} \sum_{i<j}\left(\log \left(a_{i}-a_{j}\right)^{2}+\log \frac{H^{2}\left(a_{i}-a_{j}\right)}{H\left(a_{i}-a_{j}-M\right) H\left(a_{i}-a_{j}+M\right)}\right) .
$$

The saddle-point equations are therefore

$$
\frac{8 \pi^{2} a_{i}}{\lambda}-\frac{1}{N} \sum_{i \neq j}\left(\frac{1}{a_{i}-a_{j}}-K\left(a_{i}-a_{j}\right)+\frac{1}{2}\left(K\left(a_{i}-a_{j}-M\right)+K\left(a_{i}-a_{j}+M\right)\right)\right)=0,
$$

where

$$
\begin{aligned}
K(x) & =-\frac{H^{\prime}(x)}{H(x)}=x(\psi(1+i x)+\psi(1-i x)+2 \gamma) \\
& =-2 \sum_{k=1}^{\infty}(-1)^{k} \zeta(2 k+1) x^{2 k+1},
\end{aligned}
$$

and $\psi$ is the logarithmic derivative of the $\Gamma$ function.

Introducing as usual the eigenvalue density

$$
\rho(x)=\frac{1}{N} \sum_{i} \delta\left(x-a_{i}\right)
$$


the saddle-point equation can be written as the following integral equation:

$$
f_{-\mu}^{\mu} d y \rho(y)\left(\frac{1}{x-y}-K(x-y)+\frac{1}{2}(K(x-y+M)+K(x-y-M))\right)=\frac{8 \pi^{2}}{\lambda} x .
$$

Following [24], we do the transform

$$
f_{-\mu}^{\mu} \frac{d x}{z-x} \frac{1}{\sqrt{\mu^{2}-x^{2}}} \times
$$

The integral equation then takes the form

$$
\begin{aligned}
\rho(x) & =\sqrt{\mu^{2}-x^{2}}\left(\frac{8 \pi}{\lambda}-\frac{1}{2 \pi^{2}} \int \frac{d y}{x-y} \frac{1}{\sqrt{\mu^{2}-y^{2}}}\right. \\
& \left.\times \int d z \rho(z)(2 K(y-z)-K(y-z+M)-K(y-z-M))\right) .
\end{aligned}
$$

We will treat the second term on the RHS of (4.8) as a perturbation, which allows us to replace $\rho(z)$ by $n_{0} \sqrt{\mu^{2}-z^{2}}$ where $n_{0}=2 /\left(\pi \mu^{2}\right)$ is the normalization factor. The equation can be explicitly solved in two different regimes, $\mu \ll M$ or $\mu \gg M$. Since in the perturbative approach $\mu \sim \sqrt{\lambda}$, the first case will lead to a small $\lambda$ expansion, and the second case to a strong $\lambda$ expansion. We will now consider these two cases separately.

By rescaling $x, y, z \rightarrow \mu \tilde{x}, \mu \tilde{y}, \mu \tilde{z}$, one gets new tilde variables which are of order 1 . For $\mu \ll M$ we can expand $K(\mu(\tilde{y}-\tilde{z}) \pm M)$ in powers of $(y-z)$. One has

$$
2 K(y-z)-K(y-z+M)-K(y-z-M)=-2 K^{\prime}(M)(y-z)+O\left((y-z)^{2}\right) \text {. }
$$

By computing the integrals we find

$$
\rho(x) \approx \sqrt{\mu^{2}-x^{2}}\left(\frac{8 \pi}{\lambda}-\frac{1}{\pi} K^{\prime}(M)\right) .
$$

The perturbative treatment of the saddle point equation is therefore justified for

$$
\frac{\lambda K^{\prime}(M)}{8 \pi^{2}} \ll 1
$$

The normalization condition gives

$$
\mu \approx \frac{\sqrt{\lambda}}{2 \pi \sqrt{1-\frac{\lambda K^{\prime}(M)}{8 \pi^{2}}}}
$$

The eigenvalue density (4.10) can be written in the form

$$
\rho(x) \approx \frac{8 \pi}{\lambda_{\mathrm{eff}}} \sqrt{\mu^{2}-x^{2}}
$$


with

$$
\frac{8 \pi}{\lambda_{\mathrm{eff}}} \equiv \frac{8 \pi}{\lambda}-\frac{1}{\pi} K^{\prime}(M)
$$

$K^{\prime}(M)$ is a monotonically increasing function with the behavior

$$
K^{\prime}(M) \approx \begin{cases}6 \zeta(3) M^{2}-10 \zeta(5) M^{4}, & M \ll 1 \\ 2 \log M e^{1+\gamma}-\frac{1}{6 M^{2}}, & M \gg 1\end{cases}
$$

where we have used (4.5) and the asymptotic form of the Barnes $G$ function.

$$
G(1+i M) G(1-i M) \sim \frac{e^{\frac{1}{6}}}{A^{2}} \exp \left(-\left(M^{2}+\frac{1}{6}\right) \log M+\frac{3 M^{2}}{2}+O\left(M^{-2}\right)\right) .
$$

$A$ is the Glaisher-Kinkelin constant, $A \approx 1.28$. Therefore, the condition $\mu \ll M$ together with (4.11) require $\lambda \ll 1$ for any $M$. For large $M$, one has a stronger requirement $\lambda \ll 4 \pi^{2} / \log M$.

Defining $g_{R}^{2} \equiv \lambda_{\text {eff }} / N$ and $g_{\text {bare }}^{2} \equiv \lambda / N$, for large $M$, one has

$$
\frac{4 \pi^{2}}{g_{R}^{2}}=\frac{4 \pi^{2}}{g_{\text {bare }}^{2}}-\frac{N}{2} K^{\prime}(M r) \approx \frac{4 \pi^{2}}{g_{\text {bare }}^{2}}-N \log (M r) .
$$

where we have restored the dependence on the radius $r$ of $S^{4}$. Thus, in the setup $\mathcal{N}=2^{*}$ $\mathrm{SYM}$ is interpreted as $\mathcal{N}=2 \mathrm{SYM}$ without matter multiplets with a UV regulator $M, g_{R}$ represents the renormalized gauge coupling. This interpretation leads to the familiar expression for the one-loop $\beta$ function. By replacing $M r$ by $\nu_{0} / \nu, g_{R}^{2}(\nu)$ in (4.17) represents the running gauge coupling of pure $\mathcal{N}=2 \mathrm{SYM}$, defined at an energy scale $\nu$.

Returning to the $\mathcal{N}=2^{*} \mathrm{SYM}$ theory for its own sake, we see from (4.12) that as $M$ is gradually increased at fixed $\lambda \ll 1$, the interval $(-\mu, \mu)$ of the eigenvalue distribution will expand. The approximation breaks down long before the denominator becomes singular.

We can now use the above $\rho(x)$ to calculate the expectation value of the Wilson loop operator at weak coupling. We find

$$
\begin{aligned}
W\left(C_{\text {circle }}\right) & =\int_{-\mu}^{\mu} d x \rho(x) e^{2 \pi x} \\
& =\frac{2 I_{1}\left(\sqrt{\lambda_{\text {eff }}(M)}\right)}{\sqrt{\lambda_{\text {eff }}(M)}} \\
& \approx 1+\frac{\lambda}{8}+\lambda^{2}\left(\frac{K^{\prime}(M)}{64 \pi^{2}}+\frac{1}{192}\right)+O\left(\lambda^{3}\right),
\end{aligned}
$$

or simply

$$
W\left(C_{\text {circle }}\right) \approx 1+\frac{\lambda_{\text {eff }}(M)}{8}+\frac{\lambda_{\text {eff }}^{2}(M)}{192}+O\left(\lambda_{\text {eff }}^{3}\right)
$$

Thus in this approximation the complete $M$ dependence in the Wilson loop is in $\lambda_{\text {eff }}(M)$. It would be interesting to reproduce the non-trivial $\lambda^{2}$ term proportional to $K^{\prime}(M)$ by a 
perturbative gauge theory calculation. Note that the series (4.19) has an infinite radius of convergence, since it arises from the power series expansion of the Bessel function. The approximation of course breaks down for $\lambda \sim \lambda_{0} \equiv 8 \pi^{2} / K^{\prime}(M)$, so convergence is only demonstrated at $\lambda \ll \lambda_{0}$.

Using the above expansions of $K^{\prime}(M)$, one can explicitly see the small and large $M$ behavior of the Wilson loop:

$$
\begin{aligned}
& \left.W\left(C_{\text {circle }}\right)\right|_{M \ll 1} \approx 1+\frac{\lambda}{8}+\lambda^{2}\left(\frac{3 \zeta(3) M^{2}}{32 \pi^{2}}+\frac{1}{192}\right)+O\left(\lambda^{3}\right), \\
& \left.W\left(C_{\text {circle }}\right)\right|_{M \gg 1} \approx 1+\frac{\lambda}{8}+\lambda^{2}\left(\frac{\log (M)}{32 \pi^{2}}+\frac{\gamma+1}{32 \pi^{2}}+\frac{1}{192}\right)+O\left(\lambda^{3}\right),
\end{aligned}
$$

where the second equation applies as long as $\lambda \log M \ll 1$ due to the condition (4.11).

Let us now consider the regime $\mu \gg M$. In this case we can write

$$
2 K(y-z)-K(y-z+M)-K(y-z-M)=-M^{2} K^{\prime \prime}(y-z)+O\left(M^{3}\right) .
$$

Using the rescaled variables $\tilde{y}, \tilde{z}$, it becomes clear that if $\lambda \gg 1$ the integrals will get most

of the contributions from the regions where the argument of $K^{\prime \prime}$ is large. Therefore we can approximate $K(x) \approx 2 x \log x$ so that $K^{\prime \prime}(x) \approx 2 / x$. Computing the integrals we now find

$$
\rho(x) \approx \sqrt{\mu^{2}-x^{2}} \frac{8 \pi}{\lambda}\left(1-M^{2}\right) .
$$

Therefore the perturbative approach to the saddle point equation is justified for $M^{2} \ll 1$. Now we get

$$
\mu \approx \frac{\sqrt{\lambda}}{2 \pi \sqrt{1-M^{2}}}
$$

$\mu \gg M$ requires $\lambda \gg M^{2}$. Thus we have a calculation of the circular Wilson loop in the $\mathcal{N}=2^{*}$ theory for $\lambda \gg 1$ at small $M$. In this regime, the circular Wilson loop in the $\mathcal{N}=2^{*}$ theory exhibits the following behavior

$$
W\left(C_{\text {circle }}\right) \approx e^{2 \pi \mu} \approx \exp \left[\sqrt{\lambda}\left(1+\frac{M^{2}}{2}\right)\right] .
$$

This shows that the string tension $T$ increases as a small mass parameter is turned on in the $\mathcal{N}=4$ theory, we get $T \approx \frac{\sqrt{\lambda}}{2 \pi}\left(1+\frac{M^{2}}{2}\right)$ at $M \ll 1$.

\section{$4.2 \mathcal{N}=2^{*} S U(2)$ theory}

A number of interesting properties can be derived explicitly when the gauge group is $S U(2)$. For $N=2$ the partition function reads

$$
Z^{\mathcal{N}=2^{*}}=\frac{128 \pi^{5 / 2}}{g^{3}} \int_{-\infty}^{\infty} d a a^{2} e^{-\frac{16 \pi^{2}}{g^{2}} a^{2}} \prod_{n=1}^{\infty} \frac{\left(1+\frac{4 a^{2}}{n^{2}}\right)^{2 n}}{\left(1+\frac{(2 a-M)^{2}}{n^{2}}\right)^{n}\left(1+\frac{(2 a+M)^{2}}{n^{2}}\right)^{n} e^{-\frac{2 M^{2}}{n}}}\left|Z_{\text {inst }}^{S U(2)}\right|^{2}
$$


Like in the $\mathcal{N}=2$ superconformal case, we note that in the zero-instanton sector the partition function can be written as a Laplace transform of the Borel associated function: we get

$$
\left.Z^{\mathcal{N}=2^{*}}\right|_{k=0}=\frac{2}{\sqrt{\pi \alpha}} \int_{0}^{\infty} d t e^{-t} B^{\mathcal{N}=2^{*}}(\alpha t),
$$

where

$$
\begin{aligned}
B^{\mathcal{N}=2^{*}}(x) & \equiv \sqrt{x} \frac{H^{2}(2 \sqrt{x})}{H(2 \sqrt{x}-M) H(2 \sqrt{x}+M)} \\
& =\sqrt{x} \prod_{n=1}^{\infty} \frac{\left(1+\frac{4 x}{n^{2}}\right)^{2 n}}{\left(\left(1-i \frac{M}{n}\right)^{2}+\frac{4 x}{n^{2}}\right)^{n}\left(\left(1+i \frac{M}{n}\right)^{2}+\frac{4 x}{n^{2}}\right)^{n} e^{-\frac{2 M^{2}}{n}}},
\end{aligned}
$$

and again we have defined

$$
a^{2}=\alpha t, \quad \alpha \equiv \frac{g^{2}}{16 \pi^{2}} .
$$

The associated Borel series defined by the expansion of $B^{\mathcal{N}=2^{*}}(x)$ in powers of $x$ has a finite radius of convergence determined by the first singularities that arise in $B^{\mathcal{N}=2^{*}}(x)$ at $x=\alpha t=-\frac{1}{4}(1 \pm i M)^{2}$. The associated Borel series is therefore convergent if

$$
|x|<\frac{1}{4}\left(1+M^{2}\right) .
$$

Also in this case the perturbative series has asymptotic behavior $n$ !, as follows from the fact that the series expansion of $B(x)$ around $x=0$ has a finite radius of convergence. Because the singularities lie on the complex $t$ plane, they do not affect the integration over the real positive axes of $t$. Therefore the integration is well defined (the integral is convergent) and the divergent perturbative series that one would obtain by computing Feynman diagrams is Borel summable. We have reproduced the $n$ ! asymptotic behavior by a saddle-point calculation, similarly as done above in the $\mathcal{N}=2$ superconformal case.

A systematic way to calculate the perturbative series is by writing $B^{\mathcal{N}=2^{*}}(x)$ as follows

$$
B^{\mathcal{N}=2^{*}}(x)=\sqrt{x} \frac{1}{H^{2}(M)} \exp \left[-\sum_{k=1}^{\infty} \frac{(-1)^{k}}{k}(4 x)^{k} p_{k}(M)\right],
$$

where

$$
\begin{aligned}
& p_{1}(M)=K^{\prime}(M), \\
& p_{k}(M)=2 \zeta(2 k-1)-\frac{(-1)^{k}}{(2 k-1) !} K^{(2 k-1)}(M), \quad k>1 .
\end{aligned}
$$

This form is also suitable in order to take the large $M$ limit, that we shall consider in discussing the Wilson and 't Hooft loop expectation values. For the first few terms one finds

$$
Z^{\mathcal{N}=2^{*}}=\frac{1}{H^{2}(M)}\left(1+6 p_{1} \alpha+30\left(p_{1}^{2}-p_{2}\right) \alpha^{2}+140\left(p_{1}^{3}-3 p_{1} p_{2}+2 p_{3}\right) \alpha^{3}+O\left(\alpha^{4}\right)\right) .
$$


Consider now the effects of instantons. In particular, the one-instanton contribution is given by [8]

$$
Z_{1-\mathrm{inst}}=e^{-\frac{8 \pi^{2}}{g^{2}}} \frac{2(\tilde{M}-1)^{2}\left(\tilde{M}^{2}+a^{2}-2 \tilde{M}+4\right)}{\left(4+a^{2}\right)}, \quad \tilde{M}=i M+1,
$$

where we have set the radius of $S^{4}$ to 1 . In the case $M=0$ one has $Z_{1 \text {-inst }}=0$ and one recovers the result of $\mathcal{N}=4$ theory.

Just as in the superconformal SYM case, in the one-instanton sector a new saddle point appears in the calculation of the coefficient $d_{n}$ of the $n$th term $d_{n} \alpha^{n}$ of the perturbative series, but it does not affect the convergence of the integral. We also see that the prefactor multiplying the instanton weight does not introduce singularities on the integration region which would otherwise represent an obstruction to computing the matrix integral. Indeed, the contribution (4.32) has a singularity in the complex plane at $a= \pm 2 i$ (and on the negative real axes for the integration variable $\left.t=a^{2} / \alpha\right)$. Therefore it is innocuous.

\subsubsection{Wilson loop}

Let us now consider the Wilson loop in the zero instanton sector:

$$
\left\langle e^{2 \pi q a}\right\rangle=\left(Z^{\mathcal{N}=2^{*}}\right)^{-1} \int_{-\infty}^{\infty} d a a^{2} e^{-\frac{a^{2}}{\alpha_{\mathrm{eff}}(M)}} \hat{B}^{\mathcal{N}=2^{*}}\left(a^{2}, M\right) \cosh (2 \pi q a)
$$

where we have defined (cf. (4.29) )

$$
\begin{gathered}
\hat{B}^{\mathcal{N}=2^{*}}\left(a^{2}, M\right)=\frac{1}{H^{2}(M)} \exp \left[-\sum_{k=2}^{\infty} \frac{(-1)^{k}}{k}(2 a)^{2 k} p_{k}(M)\right], \\
\frac{1}{\alpha_{\mathrm{eff}}(M)}=\frac{1}{\alpha}-4 p_{1}(M)=\frac{1}{\alpha}-4 K^{\prime}(M) .
\end{gathered}
$$

This definition of $\alpha_{\text {eff }}$ of course coincides with (4.14) when $N=2$ (it extends to any $N$ ).

In perturbation theory we obtain

$$
\left\langle e^{2 \pi q a}\right\rangle=1+3 \pi^{2} \alpha_{\mathrm{eff}} q^{2}+\alpha_{\mathrm{eff}}^{2} \frac{5 \pi^{4} q^{4}}{2}+\frac{1}{6} \pi^{2} \alpha_{\mathrm{eff}}^{3} q^{2}\left(7 \pi^{4} q^{4}-720 p_{2}\right)+O\left(\alpha_{\mathrm{eff}}^{4}\right)
$$

which exhibits the dependence on the mass parameter $M$ through $\alpha_{\text {eff }}(M)$ and $p_{2}(M)$.

One can also consider an expansion where $\alpha$ is small but $q_{*}^{2}(M) \equiv q^{2} \alpha_{\text {eff }}(M)$ is an arbitrary fixed number. We get

$$
\left\langle e^{2 \pi q a}\right\rangle=e^{\pi^{2} q_{*}^{2}}\left(1+2 \pi^{2} q_{*}^{2}-8 \pi^{2} \alpha_{\text {eff }}^{2}(M) p_{2}(M) q_{*}^{2}\left(2 \pi^{4} q_{*}^{4}+15 \pi^{2} q_{*}^{2}+15\right)+O\left(\alpha_{\text {eff }}^{3}\right)\right) .
$$

The leading term is once again the expectation value of the circular Wilson loop operator in $\mathcal{N}=4$ SYM since in the strict $\alpha \rightarrow 0$ limit with fixed $q_{*}$ the one-loop determinant is set to 1 . 
Let us apply these expressions to the study of the small and large $M$ behavior. At small $M$, one has $p_{k}(M) \approx c_{k} M^{2}$ where $c_{k}=2 k(2 k+1) \zeta(2 k+1)$. We find

$$
\left\langle e^{2 \pi q a}\right\rangle=e^{\pi^{2} q_{*}^{2}}\left(1+2 \pi^{2} q_{*}^{2}-160 \pi^{2} \zeta(5) \alpha_{\mathrm{eff}}^{2} M^{2} q_{*}^{2}\left(2 \pi^{4} q_{*}^{4}+15 \pi^{2} q_{*}^{2}+15\right)+O\left(M^{4}\right)\right)
$$

with

$$
q_{*}^{2}=q^{2} \alpha_{\mathrm{eff}}, \quad \alpha_{\mathrm{eff}} \approx \alpha+24 \zeta(3) \alpha^{2} M^{2} .
$$

We can read an $O\left(\alpha^{2} M^{2}\right)$ correction whose coefficient involves $\zeta(3)$ and $\zeta(5)$.

For large $M$ we use the following formulas

$$
\begin{aligned}
& p_{1}(M)=\ell(M)+O\left(M^{-2}\right), \quad \ell(M) \equiv 2 \log \left(M e^{\gamma+1}\right), \\
& p_{k}(M)=2 \zeta(2 k-1)+O\left(M^{-2(k-1)}\right), \quad k>1 .
\end{aligned}
$$

Thus we find

$$
\left\langle e^{2 \pi q a}\right\rangle=e^{\pi^{2} q_{*}^{2}}\left(1+2 \pi^{2} q_{*}^{2}+O\left(M^{-2}\right)\right) .
$$

Now

$$
\frac{1}{\alpha_{\mathrm{eff}}} \approx \frac{1}{\alpha}-8 \log \left(M e^{1+\gamma}\right)
$$

which can be interpreted as the expectation value of the circular Wilson loop for $\mathcal{N}=2$ SYM written in terms of the renormalized gauge coupling.

It should be noted that in the formulas (4.38), (4.41) the additional $\alpha^{3}$ corrections are suppressed, either by $M^{4}$-in the small $M$ limit- or by $M^{-2}$-in the large $M$ limit. Therefore these formulas capture the full $\alpha$ dependence if we restrict to the leading behavior in the power series expansion in the mass (or inverse mass) parameter.

\subsection{2 't Hooft loop}

The expectation value of a supersymmetric 't Hooft loop operator carrying magnetic charge, with support on the equator of $S^{4}$, is given in eq. (7.66) of [9. We set the $\theta$ parameter of the gauge coupling $\tau=\frac{\theta}{2 \pi}+\frac{4 \pi i}{g^{2}}$ to 0 and restrict to the zero instanton sector. The magnetic charges are $B=i \operatorname{diag}(p / 2,-p / 2)$ and, for concreteness, we shall consider the case of $p=$ even. Using the relations

$$
\Gamma(z) G(z)=G(1+z), \quad z \Gamma(z)=\Gamma(1+z),
$$

we find the following form for expectation value of this' $\mathrm{t}$ Hooft loop operator:

$$
\begin{aligned}
\langle T(p)\rangle & =\left(Z^{\mathcal{N}=2^{*}}\right)^{-1} \sum_{q=p, p-2, \ldots,-p} \frac{p !}{\left(\frac{p-q}{2}\right) !\left(\frac{p+q}{2}\right) !} e^{\frac{q^{2} \pi^{2}}{g^{2}}} \\
& \times \int d a e^{-\frac{16 \pi^{2} a^{2}}{g^{2}}} \frac{f_{q}(a, M) h_{p}(a, M) H^{2}(2 a)}{H(2 a-M) H(2 a+M)},
\end{aligned}
$$


where

$$
\begin{aligned}
h_{p}(a, M) & =\left(\frac{\sinh (\pi(2 a+M)) \sinh (\pi(2 a-M))}{\sinh ^{2}(2 \pi a)}\right)^{\frac{p}{2}} \\
& =\left(\frac{\cosh (4 \pi a)-\cosh (2 \pi M)}{2 \sinh ^{2}(2 \pi a)}\right)^{\frac{p}{2}}
\end{aligned}
$$

$f_{0}(a)=4 a^{2}$ and, for $q$ even, $|q| \geq 2$,

$$
f_{q}(a, M)=\frac{\left(4 a^{2}\right)^{\frac{|q|}{2}}\left(\frac{q^{2}}{4}+4 a^{2}\right)}{\left(4 a^{2}-M^{2}\right)^{\frac{|q|}{2}}} \prod_{k=1}^{\frac{|q|}{2}-1} \frac{\left(k^{2}+4 a^{2}\right)^{|q|-2 k}}{\left((k+i M)^{2}+4 a^{2}\right)^{\frac{|q|}{2}-k}\left((k-i M)^{2}+4 a^{2}\right)^{\frac{|q|}{2}-k}} .
$$

In particular,

$$
\begin{aligned}
& f_{2}(a, M)=\frac{4 a^{2}\left(1+4 a^{2}\right)}{4 a^{2}-M^{2}} \\
& f_{4}(a, M)=\frac{\left(4 a^{2}\right)^{2}\left(4 a^{2}+1\right)^{2}\left(4 a^{2}+4\right)}{\left(4 a^{2}+(1-i M)^{2}\right)\left(4 a^{2}-M^{2}\right)^{2}\left(4 a^{2}+(1+i M)^{2}\right)} .
\end{aligned}
$$

These expressions show that for any finite magnetic charge $p$ the structure of perturbation series is the same, due to the fact that poles appear only at imaginary values of $a$, not on the real axes 2 As in previous examples, the associated Borel series obtained by expanding the factor in the integrand multiplying $\exp \left[-a^{2} / \alpha\right]$ has finite radius of convergence, determined by the singularities of the integrand in the complex plane. The integration over $a$ on each term of this series produces an additional factor $n$ !. The perturbative series for $\langle T(p)\rangle$, which is again an alternate series, therefore diverges like $n$ ! but it is Borel summable because the integral over $a$ does not hit any singularity.

For $M=0$, the expression for the 't Hooft loop operator reduces to the one of $\mathcal{N}=4$ SYM theory, studied in [22,26 28]. Setting $M=0$, we see that $h_{p} \rightarrow 1$ and $f_{q}$ simplifies:

$$
f_{q}(a, M=0)=\frac{q^{2}}{4}+4 a^{2}
$$

We get $(p=2 m)$

$$
\begin{aligned}
\langle T(p)\rangle_{M=0} & =\left(Z^{\mathcal{N}=4}\right)^{-1} \sum_{n=-m}^{m}\left(\begin{array}{c}
2 m \\
m+n
\end{array}\right) e^{\frac{4 \pi^{2} n^{2}}{g^{2}}} \int d a e^{-\frac{16 \pi^{2} a^{2}}{g^{2}}}\left(n^{2}+4 a^{2}\right) \\
& =\sum_{n=-m}^{m}\left(\begin{array}{c}
2 m \\
m+n
\end{array}\right) e^{\frac{4 \pi^{2} n^{2}}{g^{2}}}\left(1+\frac{8 \pi^{2} n^{2}}{g^{2}}\right) .
\end{aligned}
$$

\footnotetext{
${ }^{2}$ Note that the poles at $2 a= \pm M$ in $f_{q}(a, M)$ are canceled by zeros of $h_{p}(a, M)$. This special point is related to the point identified in [7, where a component of the adjoint hypermultiplet is massless.
} 
Under an $S$ duality transformation $\tau \rightarrow-1 / \tau$, 't Hooft loops are mapped to Wilson loops. The precise relationship is discussed in [9]. For $\theta=0$, the $S$ duality transformation is $g \rightarrow 16 \pi^{2} / g$ and a given term in (4.48) is mapped to the $\mathcal{N}=4$ result (3.27) with $\ell=n$.

The expectation value of the 't Hooft loop operator determines the behavior of the monopole-antimonopole potential. The large loop length behavior of the 't Hooft loop operator is believed to indicate whether the theory is in a confining, Higgs or Coulomb phase. For a confining phase, the 't Hooft loop operator is expected to exhibit a perimeter law, whereas for the Higgs phase it should exhibit an area law. In the present context the 't Hooft loop is placed around the equator of $S^{4}$ and it has therefore a length proportional to $r$, the $S^{4}$ radius. Although the scale of the 't Hooft loop cannot be disentangled from the scale of $S^{4}$, it is nevertheless interesting to examine its behavior as $r$ is changed with respect to the mass parameter. In this manner by large 't Hooft loop one means $r \gg 1 / M$ or, since we have set $r=1$, it means looking at the behavior at $M \gg 1$.

Let us first expand $\langle T(p)\rangle$ in powers of $g$ (here we only study the $g \ll 1$ regime, since we are ignoring instanton contributions). At $g \ll 1$, in the sum over $q$, the terms with $q= \pm p$ dominate because of the factor $e^{\pi^{2} q^{2} / g^{2}}$. In order to study the behavior at $M \gg 1$ it is convenient to express the one-loop determinant factor in terms of $B^{\mathcal{N}=2^{*}}\left(a^{2}, M\right)$ defined in (4.34). In particular, for $p=2$, we need to compute

$$
\langle T(p=2)\rangle=\frac{2 e^{\frac{4 \pi^{2}}{g^{2}}}}{Z^{\mathcal{N}=2^{*}}} \int d a\left(1+4 a^{2}\right) \frac{4 a^{2}(\cosh (4 \pi a)-\cosh (2 \pi M))}{2 \sinh ^{2}(2 \pi a)\left(4 a^{2}-M^{2}\right)} e^{-\frac{a^{2}}{\alpha_{\text {eff }}}} \hat{B}^{\mathcal{N}=2^{*}}\left(a^{2}, M\right)
$$

$\alpha_{\text {eff }}$ has been defined in (4.35). We find

$$
\langle T(p=2)\rangle=2 e^{\frac{4 \pi^{2}}{g^{2}}}\left(T^{(1)}+\cosh (2 \pi M) T^{(2)}\right),
$$

with

$$
\begin{aligned}
T^{(1)} & =-\frac{1}{4 \pi^{2} M^{2} \alpha_{\mathrm{eff}}}-\frac{3+\left(3+5 \pi^{2}\right) M^{2}}{6 \pi^{2} M^{4}} \\
& -\frac{\alpha_{\mathrm{eff}}}{5 \pi^{2} M^{6}}\left(30 M^{4} p_{2}+\pi^{2}\left(25+\pi^{2}\right) M^{4}+5\left(3+5 \pi^{2}\right) M^{2}+15\right)+O\left(\alpha^{2}\right) \\
T^{(2)} & =\frac{1}{4 \pi^{2} M^{2} \alpha_{\mathrm{eff}}}+\frac{3-\left(\pi^{2}-3\right) M^{2}}{6 \pi^{2} M^{4}} \\
& +\frac{\alpha_{\mathrm{eff}}}{5 \pi^{2} M^{6}}\left(30 M^{4} p_{2}+\pi^{2}\left(\pi^{2}-5\right) M^{4}-5\left(\pi^{2}-3\right) M^{2}+15\right)+O\left(\alpha^{2}\right) .(4
\end{aligned}
$$

The origin of the $1 / \alpha_{\text {eff }}$ behavior in the leading term can be traced back to the fact that the integrand in the numerator now contains a term $4 a^{2}+q^{2} / 4=4 a^{2}+1$, whereas in the denominator $Z^{\mathcal{N}=2^{*}}$ contains the usual Vandermonde factor $4 a^{2}$.

In the large $M$ limit we therefore find the following behavior:

$$
\langle T(p=2)\rangle=4 e^{\frac{4 \pi^{2}}{g^{2}}} \frac{e^{2 \pi M}}{g^{2} M^{2}}\left(1-\frac{g^{2}}{2 \pi^{2}} \log M+O\left(g^{4}\right)+O\left(M^{-2}\right)\right) .
$$


This is readily extended to general $p$. For the dominant $q= \pm p$ term at $g \ll 1$ we now find

$$
\langle T(p)\rangle=c(p) \frac{1}{\left(e^{-2 \pi \operatorname{Im}(\tau)} M^{4}\right)^{\frac{p^{2}}{8}}} \frac{e^{\pi p r M}}{g^{2}}\left(1-\frac{g^{2}}{2 \pi^{2}} \log (M)+O\left(g^{4}\right)+O\left(M^{-2}\right)\right), \quad M r \gg 1,
$$

where $\tau=\frac{\theta}{2 \pi}+\frac{4 \pi i}{g^{2}} \rightarrow \frac{4 \pi i}{g^{2}}$ and we have restored the $r$ dependence.

In [7], for the theory on $\mathbf{R}^{4}$, it was pointed out that the limit to pure $N=2 \mathrm{SYM}$ theory is achieved from the $N=2^{*}$ SYM theory by taking $M$ large and holding $e^{2 \pi i \tau} M^{4}$ fixed. Strikingly, in (4.53) $\exp (2 \pi i \tau)$ appears in the right combination with the power of $M$. We now see that in our context the prescription by Seiberg and Witten has a simple physical explanation. The coupling constant dependence must be through the renormalized gauge coupling,

$$
\frac{1}{g_{R}^{2}}=\frac{1}{g^{2}}\left(1-\frac{g^{2}}{2 \pi^{2}} \log (M)\right)
$$

At large $M$, there should not be any other dependence on the bare gauge coupling which is not through the combination forming $g_{R}$. Indeed, (4.53) can be written as

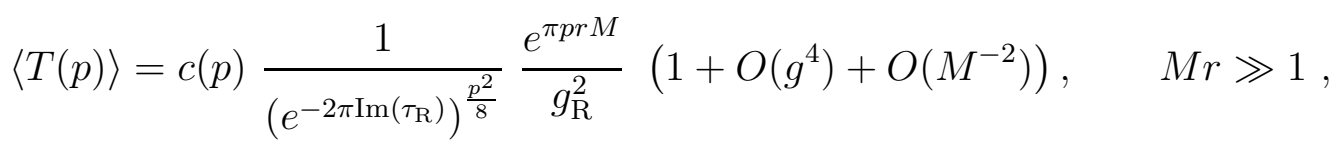

The remaining dependence on $M$ is in the exponential factor $e^{\pi p r M}$. This originates from $h_{p}$, which in turn comes from two contributions, the one-loop determinant at the equator and a contribution from monopole field configurations that screen the monopole charge $B$. Viewing $N=2^{*}$ SYM theory as pure $N=2 \mathrm{SYM}$ with a UV cutoff $M$, this factor might be indicating a very singular behavior of the expectation value of the 't Hooft loop operator in the UV limit, or it might be just reflecting the fact that the loop becomes very long and it follows a perimeter law. To clarify the meaning of this behavior it would be useful to compute the expectation value of a 't Hooft loop operator with support on a circle of arbitrary length $L$. This would allow one to consider a limit $M r \gg 1$ at fixed $L$.

\section{Concluding remarks}

One of the lessons of the matrix model examples studied here is that the divergence of the perturbation series is not inherent to the theory but rather produced by an illegitimate step in the Feynman-diagram evaluation of functional integrals, when commuting an integral with a sum: the power expansion of vertex operators with the functional integration. In this respect, the Borel summation emerges not as an artificial trick "to make convergent what is actually divergent", but it is rather built in the theory. In the simplest $S U(2)$ cases the exact gauge theory partition function automatically appears in the form of a Borel transform. It is tempting to conjecture that gauge theories are free from divergent sum 
pathologies if care is taken when commuting the functional integral with other integrals or infinite sums 3 More precisely, for certain observables the Borel function may have poles on the real axes. In this case the Borel technique requires deforming the integration contour, a procedure that is not free from ambiguities and typically produces imaginary parts. Thus, a more precise conjecture is that there should be no divergent sum pathologies in observables where an imaginary part is not expected. For observables where an imaginary part is expected (like e.g. two-point functions of unstable particles) a suitable contour deformation prescription may not only render the integral convergent but also give rise to the expected imaginary part.

To conclude, we summarize the main results.

- The perturbation series for the partition function in the $U(N) \times U(N)$ ABJM theory is an alternate asymptotic series with $(-1)^{m}(2 m) !(2 / \pi k)^{2 m}$ asymptotic behavior. This behavior is not associated with instantons (which are absent in three dimensions) but with poles on the complex plane of the one-loop determinant. The integral defining the partition function is convergent, so the divergent perturbation series is Borel summable. For the $U(2) \times U(2)$ theory, a simple integral representation illustrates the origin of the divergent perturbation series.

- For $\mathcal{N}=2 S U(2)$ SYM theory with four massless hypermultiplets, we computed the asymptotic behavior of the perturbation series for the partition function and Wilson loop operator, in the zero and one instanton sector, and again found a Borel summable perturbation series, with asymptotic behavior $(-1)^{n} n ! \alpha^{n}, \alpha=g^{2} / 16 \pi^{2}$.

- For the $\mathcal{N}=2^{*}$ theory in the planar limit, the Wilson loop at weak coupling has the same form as in $\mathcal{N}=4$ SYM theory upon replacing the 't Hooft coupling $\lambda$ by

$$
\lambda_{\text {eff }}(M)=\lambda\left(1+\frac{\lambda K^{\prime}(M)}{8 \pi^{2}}\right), \quad \frac{\lambda K^{\prime}(M)}{8 \pi^{2}} \ll 1 .
$$

At strong coupling and small mass parameter, we find $W\left(C_{\text {circle }}\right) \approx e^{2 \pi T}$ with

$$
T=\frac{\sqrt{\lambda}}{2 \pi}\left(1+\frac{M^{2}}{2}\right), \quad \lambda \gg 1, \quad M \ll 1,
$$

where $T$ can be viewed as the tension of a semiclassical string configuration. In all cases augmenting the mass parameter produces an increase of $W\left(C_{\text {circle }}\right)$. Finally, we have examined the expectation value $\langle T(p)\rangle$ of a circular 't Hooft loop operator of magnetic charge $p / 2$ in the $S U(2)$ theory. Like for the Wilson loop, the associated perturbation series in the zero instanton sector is divergent with a $(-1)^{n} n$ ! asymptotic behavior, and Borel summable. At large masses, where the theory is expected to flow to pure $\mathcal{N}=2 S U(2)$ gauge theory with a UV cutoff $M,\langle T(p)\rangle$ seems to increase as $e^{\pi p r M}$ in the weak coupling regime.

\footnotetext{
${ }^{3}$ For example, in the $U(2) \times U(2)$ ABJM model, one can compute the integral (2.26) by expanding $\mathcal{S}(v / k)$ in power series in $v / k$-which would lead to a divergent series - or by residues [18] - which would lead to a finite sum of terms.
} 
It would be interesting to study sectors of higher instanton charge in $\mathcal{N}=2 S U(2)$ SYM theories to see if more complicated instanton configurations may give rise to singularities in the Borel function lying on the integration region. This could provide an arena to clarify issues of ambiguities in contour deformation in the context of non-abelian gauge theories from closed analytic expressions.

It would also be interesting to compute observables in $N=2^{*} S U(2)$ SYM theory with a characteristic length $L$. By introducing a scale one can separate UV and IR physics in the series expansions and, in particular, see if the use of perturbation theory is problematic in the IR region, like in the case of QCD renormalons.

\section{Acknowledgements}

The author would like to thank Jaume Gomis and Mithat Ünsal for useful discussions and the Perimeter Institute for Theoretical Physics for hospitality. He acknowledges support by MCYT Research Grant No. FPA 2010-20807 and Generalitat de Catalunya under project 2009SGR502.

\section{References}

[1] F. J. Dyson, "Divergence Of Perturbation Theory In Quantum Electrodynamics," Phys. Rev. 85, 631 (1952).

[2] L. N. Lipatov, "Divergence Of The Perturbation Theory Series And The Quasiclassical Theory," Sov. Phys. JETP 45, 216 (1977) [Zh. Eksp. Teor. Fiz. 72, 411 (1977)].

[3] G. 't Hooft in The Whys of subnuclear Physics - Proceedings of the 1977 Erice Summer School. Ed. A. Zichichi (Plenum, New York, 1978).

[4] B. E. Lautrup, "On High Order Estimates In QED,” Phys. Lett. B 69, 109 (1977).

[5] M. Marino, "Nonperturbative effects and nonperturbative definitions in matrix models and topological strings," JHEP 0812, 114 (2008) arXiv:0805.3033 [hep-th]].

[6] N. Seiberg and E. Witten, "Monopole Condensation, And Confinement In N=2 Supersymmetric Yang-Mills Theory," Nucl. Phys. B 426, 19 (1994) [Erratum-ibid. B 430, 485 (1994)] arXiv:hep-th/9407087.

[7] N. Seiberg and E. Witten, "Monopoles, duality and chiral symmetry breaking in $N=$ 2 supersymmetric QCD," Nucl. Phys. B 431, 484 (1994) arXiv:hep-th/9408099.

[8] V. Pestun, "Localization of gauge theory on a four-sphere and supersymmetric Wilson loops," arXiv:0712.2824 [hep-th].

[9] J. Gomis, T. Okuda and V. Pestun, "Exact Results for 't Hooft Loops in Gauge Theories on $S^{4}, "$ arXiv:1105.2568 [hep-th]. 
[10] N. Drukker and D. J. Gross, "An exact prediction of N $=4$ SUSYM theory for string theory," J. Math. Phys. 42, 2896 (2001) arXiv:hep-th/0010274.

[11] J. K. Erickson, G. W. Semenoff and K. Zarembo, "Wilson loops in N = 4 supersymmetric Yang-Mills theory," Nucl. Phys. B 582, 155 (2000) arXiv:hep-th/0003055].

[12] O. Aharony, O. Bergman, D. L. Jafferis and J. Maldacena, "N=6 superconformal Chern-Simons-matter theories, M2-branes and their gravity duals," JHEP 0810, 091 (2008) arXiv:0806.1218 [hep-th]].

[13] A. Kapustin, B. Willett and I. Yaakov, "Exact Results for Wilson Loops in Superconformal Chern-Simons Theories with Matter," JHEP 1003, 089 (2010) arXiv:0909.4559 [hep-th]].

[14] A. Kapustin, B. Willett and I. Yaakov, "Nonperturbative Tests of Three-Dimensional Dualities," JHEP 1010, 013 (2010) [arXiv:1003.5694 [hep-th]].

[15] M. Marino and P. Putrov, "ABJM theory as a Fermi gas," arXiv:1110.4066 [hep-th].

[16] N. Drukker, M. Marino and P. Putrov, "From weak to strong coupling in ABJM theory," Commun. Math. Phys. 306, 511 (2011) arXiv:1007.3837 [hep-th]].

[17] N. Drukker, M. Marino and P. Putrov, "Nonperturbative aspects of ABJM theory," JHEP 1111, 141 (2011) arXiv:1103.4844 [hep-th]].

[18] K. Okuyama, "A Note on the Partition Function of ABJM theory on $S^{3}$," Prog. Theor. Phys. 127, 229 (2012) [arXiv:1110.3555 [hep-th]].

[19] N. A. Nekrasov, "Seiberg-Witten Prepotential From Instanton Counting," Adv. Theor. Math. Phys. 7, 831 (2004) arXiv:hep-th/0206161].

[20] N. Nekrasov and A. Okounkov, "Seiberg-Witten theory and random partitions," arXiv:hep-th/0306238.

[21] L. F. Alday, D. Gaiotto and Y. Tachikawa, "Liouville Correlation Functions from Four-dimensional Gauge Theories," Lett. Math. Phys. 91, 167 (2010) arXiv:0906.3219 [hep-th]].

[22] T. Okuda and V. Pestun, "On the instantons and the hypermultiplet mass of $N=2^{*}$ super Yang-Mills on $S^{4}, "$ arXiv:1004.1222 [hep-th].

[23] S. J. Rey and T. Suyama, "Exact Results and Holography of Wilson Loops in N=2 Superconformal (Quiver) Gauge Theories," JHEP 1101, 136 (2011) arXiv:1001.0016 [hep-th]].

[24] F. Passerini and K. Zarembo, "Wilson Loops in N=2 Super-Yang-Mills from Matrix Model," JHEP 1109, 102 (2011) [Erratum-ibid. 1110, 065 (2011)] [arXiv:1106.5763 [hep-th]]. 
[25] R. Andree and D. Young, "Wilson Loops in N=2 Superconformal Yang-Mills Theory," JHEP 1009, 095 (2010) arXiv:1007.4923 [hep-th]].

[26] J. Gomis, T. Okuda and D. Trancanelli, "Quantum 't Hooft operators and Sduality in N=4 super Yang-Mills," Adv. Theor. Math. Phys. 13, 1941 (2009) arXiv:0904.4486 [hep-th]].

[27] L. F. Alday, D. Gaiotto, S. Gukov, Y. Tachikawa and H. Verlinde, "Loop and surface operators in $\mathrm{N}=2$ gauge theory and Liouville modular geometry," JHEP 1001, 113 (2010) arXiv:0909.0945 [hep-th]].

[28] N. Drukker, J. Gomis, T. Okuda and J. Teschner, "Gauge Theory Loop Operators and Liouville Theory," JHEP 1002, 057 (2010) arXiv:0909.1105 [hep-th]]. 